\title{
Investigation of dopant centres dominating the conduction process in the bulk of un-doped GaSb
}

\author{
Megersa Wodajo Shura ${ }^{1}[$
}

Received: 22 July 2019 / Accepted: 24 September 2019 / Published online: 4 October 2019

(c) The Author(s) 2019

\begin{abstract}
In this paper, first, the theoretical description of the effects of the dopant densities and the activation energies on the ionization densities, the chemical potentials corresponding to each dopant levels, the majority carrier densities and the Fermi-energy levels in one-acceptor-level system, highly compensated system and two-acceptor-level system are described in detail. Upon fitting the theoretical to the experimental results obtained by the temperature-dependent Hall effect measurements for three samples of un-doped $\mathrm{GaSb}$, the dopant densities and the activation energies for a system with different dopants are investigated. The obtained results revealed that the dopant activation energy has less (no) effect on the Fermi-energy level and the majority carrier density in the highest temperature regimes. The doping density has also less (no) effect on the Fermienergy level in the lowest temperature regimes. Finally, fitting of the theoretical to the experimental Hall effect measurements results confirmed the presence of three acceptor and one donor levels dominating the majority carrier densities at different temperature regions in all the samples of un-doped GaSb semiconductor.
\end{abstract}

Keywords Dopant density $\cdot$ Activation energy $\cdot$ Ionization density $\cdot$ Chemical potential

\section{Introduction}

Gallium antimonide ( $\mathrm{GaSb}$ ) is a III-V semiconductor with a high charge carrier mobility, high carrier density, small and direct band gap ranging from $0.727 \mathrm{eV}$ at room temperature to $0.813 \mathrm{eV}$ at absolute zero. GaSb also has a variety of lattice parameter matched with ternary and quaternary III-V compounds $[1,2]$. Its electronic and lattice matched properties make $\mathrm{GaSb}$ a promising material for the fabrication of high-frequency optoelectronic devices working in the wide range of infrared region. In recent years, considerable needs have been developed for the fabrication of high-speed (un-cooled) infrared detectors. In optoelectronic devices, thermal transitions compete with optical ones so that high thermal generation makes non-cooled devices very noisy, thus reducing their performances at higher temperatures $[3$, 4]. Antimony-based materials were theoretically found to have the capacity to bypass these limiting phenomena $[3,4]$.

Megersa Wodajo Shura

megersawodajo@astu.edu.et

1 Department of Applied Physics, Adama Science and Technology University (ASTU), P. O. Box 1888, Adama, Ethiopia
When the temperature of an extrinsic semiconductor is increased, electrons in the valence band are transferred to the acceptor levels and the conduction band, leaving free holes behind, creating some free electrons in the conduction band and some negatively ionized states in the acceptor levels. Some electrons in the donor levels are also raised to the conduction band leaving behind some positively ionized states and creating some additional free electrons in the conduction band. At very high temperature, all the dopant levels are ionized completely and the transfer of electron from the valence band to the conduction band continues [5-7]. The densities of free carriers in the valence and the conduction bands are hence dominated by the ionization of the respective acceptor and the donor states in the lower-temperature regimes (ionization or extrinsic ranges) and by the band-toband free carriers transfer in the higher-temperature regime called (intrinsic range). When an external electric field is applied, the free charge carriers form electric current [5-7].

Several researchers have confirmed that un-doped $\mathrm{GaSb}$ is usually p-type material (majority carrier $10^{16}-10^{17} \mathrm{~cm}^{-3}$ and ionization energies $20-40 \mathrm{meV}$ ) with a residual acceptor that is doubly ionizable and associated with the Ga vacancy $[1,2$, 8-16]. Using the temperature-dependent Hall measurement and positron lifetime spectroscopy, Ling and et al. [1, 8] and 
Pödör and Somogyi [16] have reported a dominant residual acceptor level for the p-type conduction of $\mathrm{GaSb}$ (with activation energy $34 \mathrm{meV}$ and density $\sim 10^{18} \mathrm{~cm}^{-3}$ ) as most likely the GaSb antisite. Hakala et al. [2] also reported similar results with activation energy about $40 \mathrm{meV}$ in their work on self-diffusion in GaSb. Using the free carrier concentration spectroscopy, Matsuura et al. [13] also detected four acceptor levels with energies 28 to $41 \mathrm{meV}, 75$ to $99 \mathrm{meV}, 164$ to $181 \mathrm{meV}$ and $259 \mathrm{meV}$ in un-doped GaSb epilayers grown by MBE and two acceptor levels with energies $21 \mathrm{meV}$ and $83 \mathrm{meV}$ in a thick un-doped $\mathrm{GaSb}$ wafer. Hubik et al. [9, 10] reported the probability of compensation in $\mathrm{GaSb}$ (acceptor activation energy $85 \mathrm{meV}$, density $\sim 10^{17} \mathrm{~cm}^{-3}$ and donor activation energy $43 \mathrm{meV}$ and density $\sim 10^{18} \mathrm{~cm}^{-3}$ ) in their works on tellurium- and sulphur-doped GaSb using capacitance transient, Hall and photo-Hall methods. In their work on the transport properties in un-doped $p$-GaSb using PL and Hall measurements, Dutta et al. [11] have reported the possibility of self-compensation in a system with two acceptor levels (energies $30 \mathrm{meV}, 120 \mathrm{meV}$ and densities about $10^{18} \mathrm{~cm}^{-3}$ ). Zhao et al. [15] and Nakashima [12] also reported the possibility of compensation in un-doped GaSb. The work of J. S. Blakemore in the description of the majority carrier density and the Fermi-energy level in terms of the dopant density, the dopant activation energy and the temperature disregards the contribution from the intrinsic part and emphasized the contribution from the extrinsic part only [17].

This paper starts its presentation with the description of the expressions for the temperature dependences of the ionization densities and the chemical potentials corresponding to the dopant levels in terms of the doping densities, the dopant activation energies and the temperature for a system with a single dopant level. This can be done by equating the Fermi-energy level to the chemical potential corresponding to a given dopant level provided that the dopant ionization density dominates the majority carrier concentration for a system with single dopant level in the extrinsic (ionization) temperature ranges. This method can be applied to any system with more than one dopant levels, since each dopant level has its own dopant density, activation energy, chemical potential and dopant ionization. For a system with more than one acceptor/donor, the net acceptor/donor ionization density in a system is found by the summation of the ionization densities of all the acceptors/donors. The net ionization density for a compensated system is described by the difference between the net ionization densities of the acceptors and the donors. The thermal equilibrium carrier density and the Fermi-energy level for any system (compensated and multi-dopants system) can be described using the neutrality relation by introducing the net ionization density along with the contribution from the intrinsic part.

The smoothness of the curves of the temperature dependence of the majority carrier density obtained by the Hall effect measurements methods can give information about the nature of the dopants involved in the ionization of the system. The slope of the curve of the temperature dependence of the majority carrier density for a system with a single dopant level increases in a very low temperature regime, followed by a plateau at higher-temperature regime and smoothly increases in the quasi-intrinsic temperature region. For a system with more than one identical dopants, more than one bump (hill) is observed below the intrinsic region. For a compensated system, the slope of the curve increases in the lower-temperature regime and decreases in the higher-temperature regime below the quasi-intrinsic temperature region.

The nature of the curves of the temperature dependence of the majority carrier density obtained by the Hall effect measurements methods in this work reveals the presence of three acceptor levels and one donor level dominating the majority carrier density at different temperature regions in all the samples of p-type GaSb. This method cannot give information about other very deep dopants with very low densities as compared to the dominant ones.

\section{Theory}

In this section, first, we describe the general relations used to evaluate the thermal properties of both $\mathrm{p}$ - and n-type (in short $\mathrm{p} / \mathrm{n}$-types) semiconductors together. Then, the special case for the p-type material will be considered mostly in the result part. The respective thermal equilibrium hole and electron carrier densities $p_{0}$ and $n_{0}$ (in short $\left.(p / n)_{0}\right)$ in a semiconductor are described by $[5,6,17,18]$ :

$(p / n)_{0}=N_{\mathrm{V} / \mathrm{C}} \exp \left((-/+) \frac{E_{\mathrm{F}}-E_{\mathrm{V} / \mathrm{C}}}{k_{\mathrm{B}} T}\right)$,

where $E_{\mathrm{F}}$ is the Fermi energy, $E_{\mathrm{V} / \mathrm{C}}$ the maximum/minimum valence/conduction band energy, $k_{\mathrm{B}}$ the Boltzmann constant, $T$ the temperature of the system, and $N_{\mathrm{V} / \mathrm{C}}$ is the effective density of the valence/conduction band states given by:

$N_{\mathrm{V} / \mathrm{C}}=2\left(\frac{2 \pi m_{\mathrm{p} / \mathrm{n}}^{*} k_{\mathrm{B}} T}{h^{2}}\right)^{3 / 2}$,

where $h$ is Planck's constant and $m_{\mathrm{p} / \mathrm{n}}^{*}$ is the hole/electron density-of-states effective masses in the valence/conduction band. For p-type GaSb, $m_{\mathrm{p}}^{*}=0.35 m_{\mathrm{n}}$ and $m_{\mathrm{n}}^{*}=0.047 m_{\mathrm{n}}$, where $m_{\mathrm{n}}$ is the electron rest mass. The intrinsic carrier density can be described from the product of $p_{0}$ and $n_{0}$ in relation (1) as:

$n_{i}=\sqrt{p_{0} n_{0}}=\sqrt{N_{\mathrm{C}} N_{\mathrm{V}}} \exp \left(-\frac{E_{\mathrm{g}}}{2 k_{\mathrm{B}} T}\right)$, 
where $E_{\mathrm{g}}$ is the energy band gap between $E_{\mathrm{C}}$ and $E_{\mathrm{V}}$. Relation (3) is called the law of mass action.

The first empirical relation for the band gap shifts with temperature was developed by Varshini et al. [5-7, 19] as:

$E_{\mathrm{g}}(T)=E_{\mathrm{g} 0}-\frac{\alpha T^{2}}{T+\beta}$,

where $\alpha$ and $\beta$ are constants with values $94 \mathrm{~K}$ and $3.78 \times 10^{-4} \mathrm{eV} / \mathrm{K}$, respectively, and $E_{\mathrm{g} 0}$ is the limiting value of the band gap at absolute zero which is equal to $0.813 \mathrm{eV}$ for $\mathrm{GaSb}$. The variations of $E_{\mathrm{V}}$ and $E_{\mathrm{C}}$ with temperature can be described using relations (4) by keeping the mid-gap at one half of $E_{\mathrm{g} 0}$ at all temperatures.

The density of the ionized acceptor/donor $N_{\mathrm{a} / \mathrm{d}}^{(-/+)}$, can be given in terms of the respective density of the acceptor/donor, $N_{\mathrm{a} / \mathrm{d}}$, as $[5,6,17,18]$ :

$N_{\mathrm{a} / \mathrm{d}}^{(-/+)}=\frac{N_{\mathrm{a} / \mathrm{d}}}{1+g_{\mathrm{a} / \mathrm{d}} \exp \left((+/-)\left(\frac{E_{\mathrm{a} / \mathrm{d}}-E_{\mu(a / d)}}{k_{\mathrm{B}} T}\right)\right)}$,

where $E_{\mu(\mathrm{a} / \mathrm{d})}$ represents the chemical potential corresponding to the acceptor/donor and $E_{\mathrm{a} / \mathrm{d}}$ represents the energy level of the acceptor/donor level, $g_{\mathrm{a} / \mathrm{d}}$ represents the acceptor/donor degeneracy factor reflecting the degeneracy of the valence/ conduction with $g_{\mathrm{d}}=2$ for one parabolic band at the conduction band edge and $g_{\mathrm{a}}=4$ for the twofold degeneracy of the valence band (heavy and light holes). If a total of $n$ acceptors/donors are involved in the ionization of the system, the net acceptor/donor ionization density is given by the sum of the ionization densities, $N_{(\mathrm{a} / \mathrm{d}) i}^{(-/+)}$, of the corresponding acceptors/donors:

$N_{\mathrm{a} / \mathrm{d}}^{(-/+)}=\sum_{i=1}^{n} N_{(\mathrm{a} / \mathrm{d}) i}^{(-/+)}$.

Taking into account the well-known neutrality relation [1] along with the law of mass action in relation (3), we get:

$(p / n)_{0}=\frac{N_{\mathrm{a} / \mathrm{d}}^{(-/+)}-N_{\mathrm{d} / \mathrm{a}}^{(+/-)}}{2}+\sqrt{n_{i}^{2}+\left(\frac{N_{\mathrm{a}}^{-}-N_{\mathrm{d}}^{+}}{2}\right)^{2}}$.

If the net acceptor density, $N_{\mathrm{a}}$, and the net donor density, $N_{\mathrm{d}}$, are equal, the material is said to be completely (highly) compensated. Since the ionized densities, $N_{\mathrm{a} / \mathrm{d}}^{(-/)}$, are affected by several factors, the acceptor ionization density, $N_{\mathrm{a}}^{-}$, in the highly compensated material might not be equal to the donor ionization density, $N_{\mathrm{d}}^{+}$, as we shall describe later.

\section{Methodology}

The three samples used in this study were un-doped epitaxial p-type GaSb grown in Nelson Mandela Metropolitan University Physics laboratory by metal-organic vapour phase epitaxy (MOVPE) on semi-insulating (001) GaAs substrate. The data of the majority carrier densities between 10 and $300 \mathrm{~K}$ are obtained using normal temperature-dependent Hall effect measurements. The data of the majority carrier densities between 300 and $400 \mathrm{~K}$ are obtained using modified temperature-dependent Hall effect measurement setup. The thicknesses of the samples are $1.8 \mu \mathrm{m}, 3.7 \mu \mathrm{m}$ and $15.3 \mu \mathrm{m}$, and the typical thermal equilibrium majority carrier concentrations are $2.15 \times 10^{17} \mathrm{~cm}^{-3}, 1.45 \times 10^{17} \mathrm{~cm}^{-3}$ and $4.2 \times 10^{16} \mathrm{~cm}^{-3}$, respectively. The results for the majority carrier densities obtained using the Hall effect measurements were obtained by heating and cooling the sample between the temperatures of $10 \mathrm{~K}$ and $400 \mathrm{~K}$.

\section{Results and discussion}

In this section, first, the preliminary concepts of the effects of the dopant density and activation energy on the ionization density and chemical potential corresponding to the given dopant level are evaluated in detail. Then, the temperature dependence of the dopant ionization densities, the chemical potential corresponding to the dopant levels and the majority carrier densities and the Fermi-energy level for one-acceptor-level system, for a highly compensated system and for two-acceptor-level system are described using illustrations. Finally, the fitting of the experimental results of the majority carrier densities and the Fermi-energy levels with the theoretical results will be performed. The dopant densities and the corresponding activation energies of the dopants are determined from the fitting results.

\section{Preliminary concepts}

For material with one acceptor/donor level, relation (7) is simplified to:

$(p / n)_{0}=\frac{N_{\mathrm{a} / \mathrm{d}}^{(-/+)}}{2}+\sqrt{n_{i}^{2}+\left(\frac{N_{\mathrm{a} / \mathrm{d}}^{(-/+)}}{2}\right)^{2}}$.

The net ionization density, $N_{\mathrm{a} / \mathrm{d}}^{(-/+)}$, and the intrinsic carrier concentration, $n_{i}$, in relation (8) are competing. In the higher-temperature regimes called intrinsic temperature range, the net ionization density is very small $\left(N_{\mathrm{a} / \mathrm{d}}^{(-/+)} \ll n_{i}\right)$ and the majority carrier density concentration, $(p / n)_{0}$, is 
equal to $n_{i}$. In the intermediate-temperature regimes between the intrinsic temperature range called quasi-intrinsic temperature range, both $N_{\mathrm{a} / \mathrm{d}}^{(-/+)}$and $n_{i}$ are dominating the entire majority carrier concentration $(p / n)_{0}$ as in relation (8). In the lower-temperature range called extrinsic temperature range below the quasi-intrinsic temperature range, the intrinsic carrier concentration, $n_{i}$, is very small $\left(N_{\mathrm{a} / \mathrm{d}}^{(-/+)} \gg n_{i}\right)$ and the majority carrier concentration, $(p / n)_{0}$, is equal to $N_{\mathrm{a} / \mathrm{d}}^{(-/+)}$. Using this information along with relation (3), one can show for extrinsic semiconductor that:

$\frac{n_{i}}{(n / p)_{0}}=\frac{(p / n)_{0}}{n_{i}}=\frac{N_{\mathrm{a} / \mathrm{d}}^{(-/+)}}{n_{i}}>1$.

This shows that the addition of different acceptor and donor impurity concentrations to the semiconductor increases the majority carrier density above the intrinsic level $\left((p / n)_{0}>n_{i}\right)$ and decreases the minority carrier density below the intrinsic level $\left((n / p)_{0}<n_{i}\right)$.

For extrinsic semiconductor with one acceptor/donor level, the ionized acceptor/donor density, $N_{\mathrm{a} / \mathrm{d}}^{(-/+)}$, dominates the entire majority carrier density, $(p / n)_{0}$, as in relation (9). The chemical potential corresponding to this acceptor/ donor $E_{\mu(a / d)}$ also dominates the entire Fermi energy, $E_{\mathrm{F}}$, in the lower-temperature regimes. Thus, upon replacing the majority carrier concentration $(p / n)_{0}$ by $N_{\mathrm{a} / \mathrm{d}}^{(-+)}$[17] and $E_{\mathrm{F}}$ by $E_{\mu(\mathrm{a} / \mathrm{d})}$ in the extrinsic region, relation (1) can be modified under this condition as:

$N_{\mathrm{a} / \mathrm{d}}^{(-/+)}=N_{\mathrm{V} / \mathrm{C}} \exp \left((-/+) \frac{E_{\mu(\mathrm{a} / \mathrm{d})}-E_{\mathrm{V} / \mathrm{C}}}{k_{\mathrm{B}} T}\right)$.

Eliminating $E_{\mu(\text { a/d) }}$ between relations (5) and (10) gives the density of the ionized dopants as:

$$
N_{\mathrm{a} / \mathrm{d}}^{(-/+)}=\frac{2 N_{\mathrm{a} / \mathrm{d}}}{1+\sqrt{1+\frac{4 g_{\text {add }} N_{\text {a/d }}}{N_{\mathrm{V} / \mathrm{C}}} \exp \left(\frac{\Delta E_{\text {a/d }}}{k_{\mathrm{B}} T}\right)}},
$$

where $\Delta E_{\mathrm{a} / \mathrm{d}}$ is the acceptor/donor activation energy given by $(+/-)\left(E_{\mathrm{a} / \mathrm{d}}-E_{\mathrm{V} / \mathrm{C}}\right)$. Again, upon eliminating $N_{\mathrm{a} / \mathrm{d}}^{(-/+)}$between relations (5) and (10), we obtain the chemical potential $E_{\mu(\mathrm{a} / \mathrm{d})}$ corresponding to the acceptor/donor level as:

$$
\begin{aligned}
E_{\mu(\mathrm{a} / \mathrm{d})}= & E_{\mathrm{V} / \mathrm{C}}+/-k_{\mathrm{B}} T \\
& \times \ln \left(\frac{N_{\mathrm{V} / \mathrm{C}}}{2 N_{\mathrm{a} / \mathrm{d}}}\left[1+\sqrt{1+\frac{4 g_{\mathrm{a} / \mathrm{d}} N_{\mathrm{a} / \mathrm{d}}}{N_{\mathrm{V} / \mathrm{C}}} \exp \left(\frac{\Delta E_{\mathrm{a} / \mathrm{d}}}{k_{\mathrm{B}} T}\right)}\right] .\right.
\end{aligned}
$$

The only variable on the right sides of relations (11) and (12) is the temperature. As we shall see in the next section, the variation of the ionization energies $\Delta E_{\mathrm{a} / \mathrm{d}}$ with temperature does not affect the results of $N_{\mathrm{a} / \mathrm{d}}^{(-/+)}$and $E_{\mu(\mathrm{a} / \mathrm{d})}$. Thus, the two relations (11) and (12) are very suitable for the modelling of the temperature dependences of $N_{\mathrm{a} / \mathrm{d}}^{(-/+)}, E_{\mu(\mathrm{a} / \mathrm{d})}$, $(p / n)_{0}$ and $E_{\mathrm{F}}$ as we shall describe the details for different cases later.

The dopant densities are very much less than the effective densities of the band's states $\left(N_{\mathrm{a} / \mathrm{d}} \ll N_{\mathrm{V} / \mathrm{C}}\right)$. Therefore, the dopant activation energies ( $\Delta E_{\mathrm{a} / \mathrm{d}}$ can be very much greater than $k_{\mathrm{B}} T$ (or $\Delta E_{\mathrm{a} / \mathrm{d}} \gg k_{\mathrm{B}} T$ ) at very low temperature. Hence, the second terms in the square roots of relations (11) and (12) are very much greater than 1 at very low temperature. Again, $\Delta E_{\mathrm{a} / \mathrm{d}}$ can be very much less than $k_{\mathrm{B}} T\left(\Delta E_{\mathrm{a} / \mathrm{d}} \ll k_{\mathrm{B}} T\right)$ at very high temperature and the second terms in the square roots of relations (11) and (12) are very much less than 1. The dopant ionizations and the corresponding chemical potentials have hence the following two limiting cases:

$N_{\mathrm{a} / \mathrm{d}}^{(-+)}= \begin{cases}\sqrt{\frac{N_{\mathrm{a} / \mathrm{d}} N_{\mathrm{V} / \mathrm{c}}}{g_{\text {a/d }}}} \exp \left(-\frac{\Delta E_{\mathrm{a} / \mathrm{d}}}{2 k_{\mathrm{B}} T}\right) & \text { low } T, \\ N_{\mathrm{a} / \mathrm{d}}, & \operatorname{high} T\end{cases}$

and

$E_{\mu(\mathrm{a} / \mathrm{d})}=\left\{\begin{array}{lr}E_{\mathrm{V} / \mathrm{C}}+/-\left[\frac{\Delta E_{\mathrm{add}}}{2}+\frac{k_{\mathrm{B}} T}{2} \ln \left(\frac{g_{\mathrm{ad}} N_{\mathrm{V} / \mathrm{C}}}{N_{\mathrm{a} / \mathrm{d}}}\right)\right], & \operatorname{low} T, \\ E_{\mathrm{V} / \mathrm{C}}+/-k_{\mathrm{B}} T \ln \left(\frac{N_{\mathrm{V} / \mathrm{C}}}{N_{\mathrm{a} / \mathrm{d}}}\right), & \operatorname{high} T .\end{array}\right.$

The ionization energies, $\Delta E_{\mathrm{a} / \mathrm{d}}$, affect the values of the dopant ionization densities, $N_{\mathrm{a} / \mathrm{d}}^{(-/+)}$, and the corresponding chemical potentials, $E_{\mu(\mathrm{a} / \mathrm{d})}$, only in the very low temperature regimes closer to absolute zero as can be seen in relations (13) and (14). Thus, keeping the values of the ionization energies, $\Delta E_{\mathrm{a} / \mathrm{d}}$, constant during the analysis does not affect the results of the temperature dependence of the dopant ionization densities, $N_{\mathrm{a} / \mathrm{d}}^{(-/)}$, and the chemical potentials, $E_{\mu(\mathrm{a} / \mathrm{d})}$, as predicted at the end of the previous section.

One can see from relation (13) that the dopant ionization densities increase with increasing temperature and decreasing the dopant activation energies in the lower-temperature regime and are unaffected by both the temperature and the dopant activation energies in the higher-temperature regime. As one can see from relation (14), the term in the square bracket with temperature coefficients approaches zero at very low temperature. Thus, the depth of the chemical potentials corresponding to the dopant levels remains constant at one half of the activation energies and less (not) affected by the temperature and the dopant densities at very low temperature regimes. The very high temperature chemical potentials corresponding to the dopant levels increase in depth with increasing the temperature and decreasing the dopant densities and are unaffected by the dopant activation energies. 


\section{System with one acceptor level}

Figure 1 shows the temperature dependence of (a) the acceptor ionization density (magenta line), (b) the majority (wine line), minority (green line) and intrinsic (dark yellow line) carrier densities along with the acceptor ionization density (magenta line) and (c) the energy band structure including valence band energy (black line), conduction band energy (black line), Fermi level (wine line), intrinsic energy (dark yellow line) and chemical potential corresponding the acceptor (magenta line) for a p-type GaSb semiconductor of acceptor density $10^{16} \mathrm{~cm}^{-3}$ and activation energy of $1 \mathrm{meV}$.

The variation of $N_{\mathrm{a}}^{-}$with temperature depicted in Fig. 1a is described using relations (11). Based on the limiting values of temperature ranges of $N_{\mathrm{a}}^{-}$in relation (13), the area
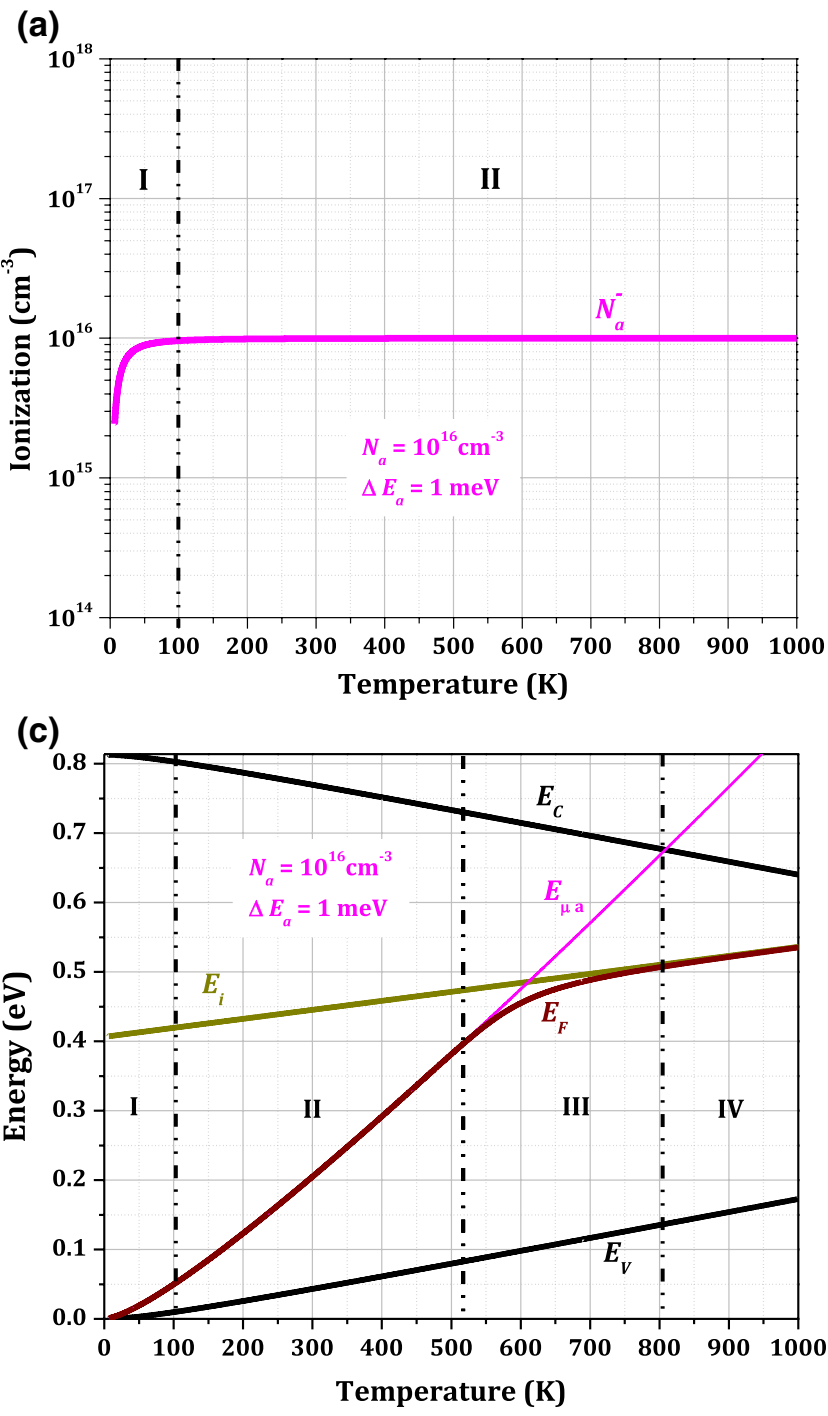

Fig. 1 Temperature dependence of a the acceptor ionization density, b the majority, minority and intrinsic carrier densities along with the acceptor ionization density and $\mathbf{c}$ the energy band structure of p-type under Fig. 1a is divided into two regions. Region $\mathrm{I}$ is a lower-temperature region in which $N_{\mathrm{a}}^{-}$is increasing with temperature as predicted in relation (13) and is known as partial ionization region. Region II is the higher-temperature range in which the acceptor level is completely ionized to $N_{\mathrm{a}}$ $\left(N_{\mathrm{a}}=10^{16} \mathrm{~cm}^{-3}\right.$ in this case), and hence it is called complete ionization region.

The resulting majority carrier concentration, $p_{0}$, is obtained directly by substituting relations (3) and (11) into relation (7) for very low density of the donor state $\left(N_{\mathrm{a}} \rightarrow 0\right)$. The minority carrier density, $n_{0}$, is then determined using the law of mass action relation (3). The majority carrier density, $p_{0}$, depicted in Fig. $1 \mathrm{~b}$ shows similar properties with that of $N_{\mathrm{a}}^{-}$in the partial ionization region (Region I) and the lower-temperature regimes of the complete ionization (b)

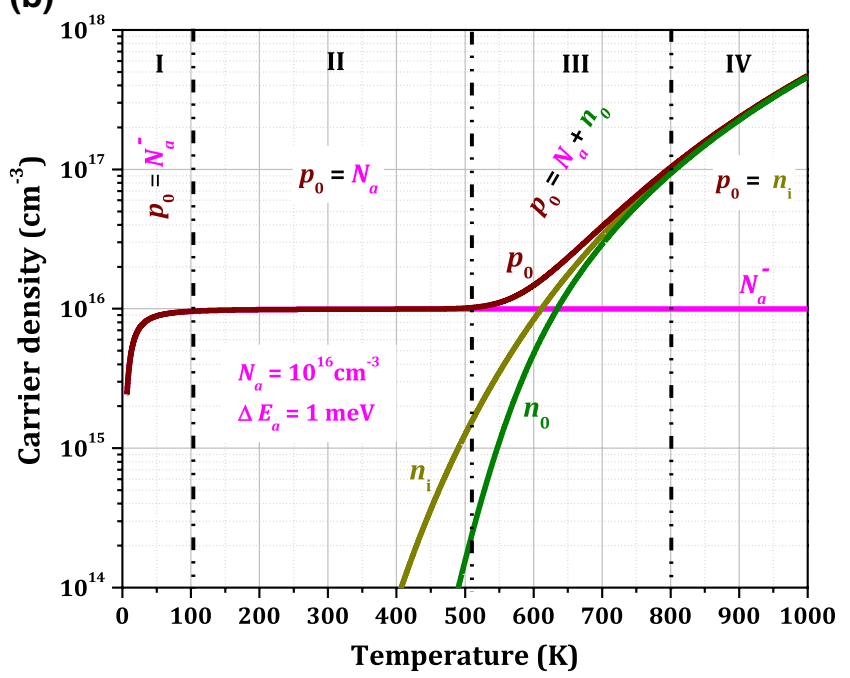

GaSb semiconductor of acceptor density $10^{16} \mathrm{~cm}^{-3}$ and acceptor energy level of $1 \mathrm{meV}$ 
region (Region II) of Fig. 1a. Because of the dominance of the intrinsic carrier density, $n_{i}$ over the acceptor state density, $N_{\mathrm{a}}$ in the very high temperature regimes, the majority carrier density, $p_{0}$, is started to increase with increasing the temperature in the higher-temperature regimes of Regions II of Fig. 1a. Therefore, the majority carrier density, $p_{0}$, in Fig. $1 \mathrm{~b}$ has the following four limiting cases:

$p_{0}= \begin{cases}\sqrt{\frac{N_{\mathrm{a}} N_{\mathrm{V}}}{g_{\mathrm{a}}}} \exp \left(-\frac{\Delta E_{\mathrm{a}}}{2 k_{\mathrm{B}} T}\right), & \text { in Region I, } \\ N_{\mathrm{a}}, & \text { in Region II, } \\ \frac{N_{\mathrm{a}}}{2}+\sqrt{n_{i}^{2}+\frac{N_{\mathrm{a}}^{2}}{4},} & \text { in Region III, } \\ n_{i}, & \text { in Region IV. }\end{cases}$

Based on relation (15), the area under the curve of $p_{0}$ in Fig. $1 b$ is then divided into four regions. Region I of Fig. 1b is the region where the partial ionization dominates the entire majority carrier $p_{0}$. This region is also corresponding to Region I in Fig. 1a, and hence $p_{0}$ is equal to $N_{\mathrm{a}}^{-}$. Region II in Fig. $1 \mathrm{~b}$ is the region where the complete ionization region dominates the entire majority carrier density, $p_{0}$, and hence $p_{0}$ is equal to $N_{\mathrm{a}}$. Since Regions I and II are controlled by the acceptor dopant density, they are categorized in the extrinsic region described under "Preliminary concepts" section. Region III under the curve of $p_{0}$ in Fig. $1 \mathrm{~b}$ is the region in which both the acceptor ionization and the intrinsic carrier densities dominate the total majority carrier density, $p_{0}$. The majority carrier density, $p_{0}$, in this region can be given by the sum of $N_{\mathrm{a}}$ and the minority carrier density, $n_{0}$. Region III is the range of temperature for the transition between the extrinsic and intrinsic regions and hence called quasiintrinsic region. Region IV is the region in which the effect of acceptor ionization is negligible and both the majority and minority carrier densities are equal to the intrinsic concentration. Hence, Region IV is called intrinsic region.

The temperature dependence of the chemical potential for a system with one acceptor level shown in Fig. 1c is obtained as a function of temperature using relation (12) by keeping $N_{\mathrm{a}}$ and $\Delta E_{\mathrm{a}}$ constant and varying temperature $T$. The intrinsic energy level, $E_{\mathrm{i}}$, can be determined in this case from relation (1) by changing $p_{0}$ and $n_{0}$ to $n_{i}$ and $E_{\mathrm{F}}$ to $E_{\mathrm{i}}$. The Fermi-energy level, $E_{\mathrm{F}}$, can be evaluated in general using relation (7) along with relation (1). The value of the chemical potential, $E_{\mu \mathrm{a}}$, corresponding to the acceptor level has lower value at very low temperature regimes (near the absolute zero) of Region I and increases with increasing temperature as predicted by the second part of relation (14).

When the limiting values $p_{0}$ in relation (15) are applied to relation (1), the Fermi-energy level, $E_{\mathrm{F}}$, is found to be:
$E_{\mathrm{F}}= \begin{cases}E_{\mathrm{V}}+\frac{\Delta E_{\mathrm{a}}}{2}+\frac{k_{\mathrm{B}} T}{2} \ln \left(\frac{g_{\mathrm{a}} N_{\mathrm{V}}}{N_{\mathrm{a}}}\right), & \text { in Region I, } \\ E_{\mathrm{V}}+k_{B} T \ln \left(\frac{N_{\mathrm{V}}}{N_{\mathrm{a}}}\right), & \text { in Region II, } \\ E_{\mathrm{V}}+k_{\mathrm{B}} T \ln \left(\frac{2 N_{\mathrm{V}}}{N_{\mathrm{a}}+\sqrt{4 n_{i}^{2}+N_{\mathrm{a}}^{2}}}\right), & \text { in Region III, } \\ E_{\mathrm{i}}, & \text { in Region IV. }\end{cases}$

Based on relation (16), the area under the curve of $E_{\mathrm{F}}$ in Fig. 1c can also be divided into four regions I, II, III and IV that are identical to the corresponding regions in Fig. 1b. In Regions I and II (extrinsic regions) of Fig. 1c, the chemical potential, $E_{\mu \mathrm{a}}$, corresponding to the given acceptor level dominates the entire Fermi level, $E_{\mathrm{F}}$, of the system. In Region III (quasi-intrinsic region), both the chemical potential, $E_{\mu \mathrm{a}}$, corresponding to the given acceptor and the intrinsic energy, $E_{\mathrm{i}}$, dominate the entire Fermi-energy level, $E_{\mathrm{F}}$. Region IV under the curves in Fig. 1c also corresponds to the intrinsic region (Region IV) of Fig. 1b. The intrinsic energy, $E_{\mathrm{i}}$, dominates the entire Fermi-enrgy level, $E_{\mathrm{F}}$, in Region IV of Fig. 1c. The chemical potential, $E_{\mu \mathrm{a}}$, has influence on the Fermi-energy level, $E_{\mathrm{F}}$, only in Regions I, II and III of Fig. 1c. Even though the value of the chemical potential, $E_{\mu \mathrm{a}}$, beyond the quasi-intrinsic region has no contribution to the Fermi-energy level, the curve of $E_{\mu \mathrm{a}}$ in Region IV of Fig. 1c is drawn to lead the direction of the variation of $E_{\mu \mathrm{a}}$ in the higher-temperature regimes. The boundaries of all the regions under the curves in Fig. 1a-c are not clearly defined in this work.

\section{Highly compensated system}

Figure 2 illustrates the temperature dependence of (a) $N_{\mathrm{a}}^{-}$ (magenta line), $N_{\mathrm{d}}^{+}$(blue line) and $N_{\mathrm{a}}^{-}-N_{\mathrm{d}}^{+}$(red line), (b) $p_{0}$ (wine line), $n_{i}$ (green line) and $n_{i}$ (yellow line) along with $N_{\mathrm{a}}^{-}-N_{\mathrm{d}}^{+}$(red line) and (c) the energy band structure consisting of $E_{\mathrm{V}}$ and $E_{\mathrm{C}}$ (black lines), $E_{\mathrm{F}}$ (wine line), $E_{\mathrm{i}}$ (yellow line), $E_{\mu \mathrm{a}}$ (magenta line) and $E_{\mu \mathrm{d}}$ (blue line) for a highly compensated semiconductor of equal acceptor and donor densities of $10^{17} \mathrm{~cm}^{-3}$ and equal acceptor and donor activation energies of $1 \mathrm{meV}$. The net ionization density is obtained by subtracting $N_{\mathrm{d}}^{+}$from $N_{\mathrm{a}}^{-}$in relation (11). The majority carrier density, $p_{0}$, is described using relation (7) by taking into account the net ionization condition with $N_{\mathrm{a}}=N_{\mathrm{d}}$. The other quantities are described in the same way as discussed in the previous sections.

As can be seen in Fig. 2a, in a highly compensated p-type $\operatorname{GaSb}\left(N_{\mathrm{a}}=N_{\mathrm{d}}\right)$ with equal acceptor and donor activation 


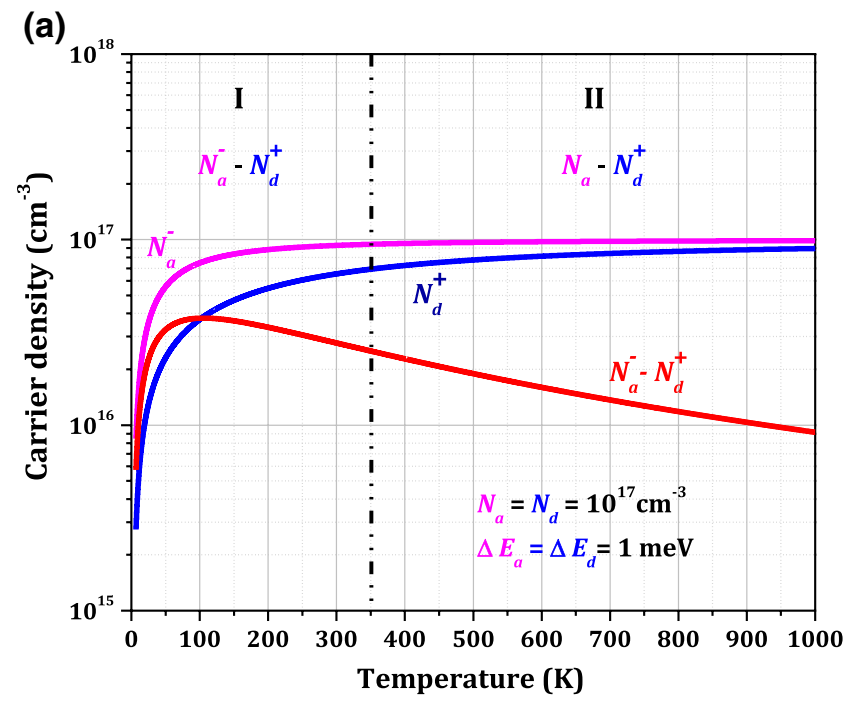

(c)

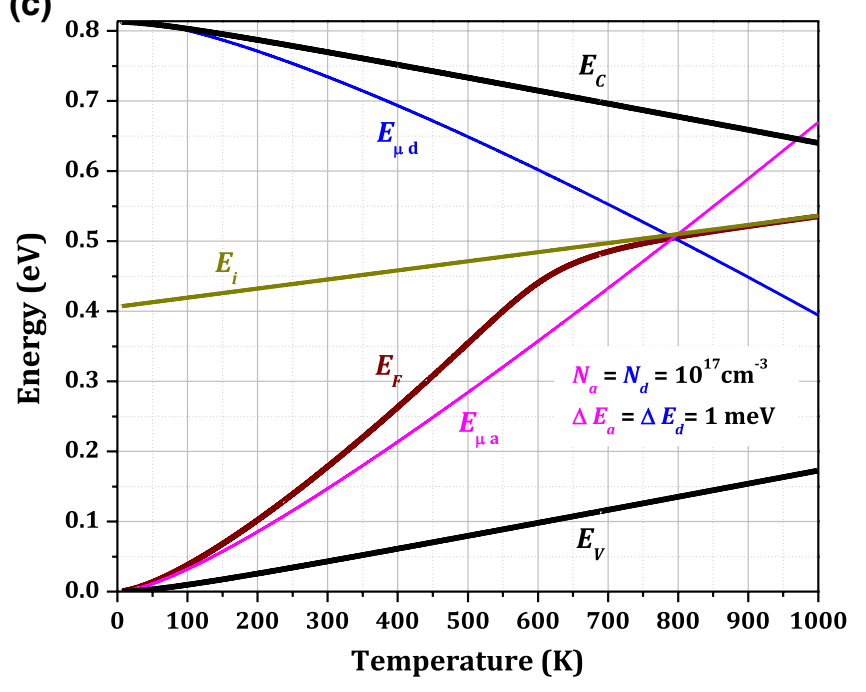

Fig. 2 Temperature dependence of a $N_{\mathrm{a}}^{-}$(magenta line), $N_{\mathrm{d}}^{+}$(blue line) and $N_{\mathrm{a}}^{-}-N_{\mathrm{d}}^{+}$(red line), $\mathbf{b} p_{0}$ (wine line), $n_{0}$ (green line) and $n_{i}$ (yellow line) along with $N_{\mathrm{a}}^{-}-N_{\mathrm{d}}^{+}$(red line) and $\mathbf{c}$ the energy band structure consisting of $E_{\mathrm{V}}$ and $E_{\mathrm{C}}$ (black lines), $E_{\mathrm{F}}$ (wine line), $E_{\mathrm{i}}$ (yel-

energies $\left(\Delta E_{\mu \mathrm{a}}=\Delta E_{\mu \mathrm{d}}\right)$, the donor ionization density is less than the acceptor ionization density $\left(N_{\mathrm{d}}^{+}<N_{\mathrm{a}}^{-}\right)$in the lowertemperature regime. The acceptor ionization density, $N_{\mathrm{a}}^{-}$, also reaches the complete ionization faster as compared to the donor ionization density, $N_{\mathrm{d}}^{+}$. This variation is attributed to the fact that, in the lower-temperature regimes, the effective density of the conduction band states $\left(N_{\mathrm{C}}\right)$ that is highly influencing $N_{\mathrm{d}}^{+}$[see relation (14)] is smaller than the effective density of the valence band states $\left(N_{\mathrm{V}}\right)$ that is influencing $N_{\mathrm{a}}^{-}$[see relation (13)]. The difference in the values of the degeneracy of the valence and conduction bands $\left(g_{\mathrm{a}}\right.$ and $\left.g_{\mathrm{d}}\right)$ can also affect the ionization densities at the lower-temperature regimes [see relation (13)]. The two regions I and II in

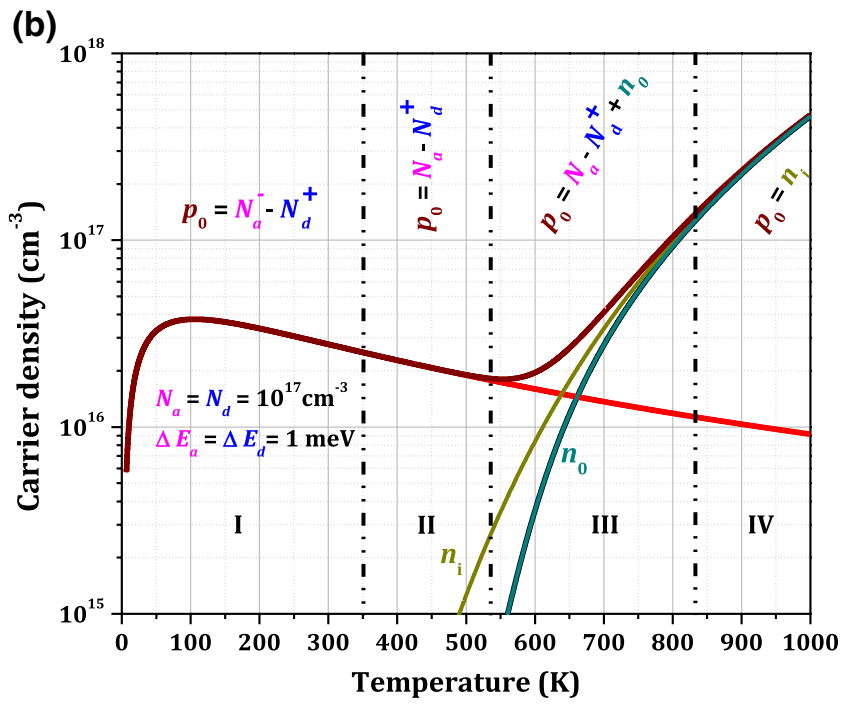

low line), $E_{\mu \mathrm{a}}$ (magenta line) and $E_{\mu \mathrm{d}}$ (blue line) for a highly compensated semiconductor of equal acceptor and donor densities of $10^{17} \mathrm{~cm}^{-3}$ and equal acceptor and donor activation energies of $1 \mathrm{meV}$

Fig. 1a are also suitable for the description of the properties of the regions under the compensated system in Fig. 2a. Region I of Fig. 2a is a lower-temperature region where both the acceptor and donor levels are partially ionized and the net ionization is given by $N_{\mathrm{a}}^{-}-N_{\mathrm{d}}^{+}$. As a result, the net ionization density increases with increasing temperature in the lower-temperature regimes of Region I where $N_{\mathrm{a}}^{-} \gg N_{\mathrm{d}}^{+}$and decreases with temperature in the higher-temperature regimes of Region I where $N_{\mathrm{d}}^{+}$started to influence the net ionization density. In Region II, the acceptor level is completely ionized and the donor level is still under partial ionization, so that the net ionization becomes $N_{\mathrm{a}}-N_{\mathrm{d}}^{+}$and decreases with increasing $N_{\mathrm{d}}^{+}$as shown by red line in Fig. 2a. The partial ionization region gets wider in the donor level 
than the acceptor level in highly compensated p-type GaSb. Therefore, the larger the effective density of state of a given band, the larger the corresponding ionization density influenced by the band and the faster it reaches the complete ionization region.

Since the net ionization dominates the majority carrier density in the lower-temperature regimes, $p_{0}$ also increases with increasing temperature in the lower-temperature regime of Region I where $p_{0} \approx N_{\mathrm{a}}^{-} \gg N_{\mathrm{d}}^{+}$and decreases with increasing temperature in the relatively higher-temperature regimes of Region I where $N_{\mathrm{d}}^{+}$is influencing the entire net ionization density. In region II where the acceptor levels are completely ionized, $p_{0} \approx N_{\mathrm{a}}-N_{\mathrm{d}}^{+}$and hence decreases with increasing temperature as shown in Fig. $2 \mathrm{~b}$ until the system reaches the quasi-intrinsic region (Region III). In the quasineutral region (Region III), the minority carrier also contributes to the majority carrier density and $p_{0} \approx N_{\mathrm{a}}-N_{\mathrm{d}}^{+}+n_{0}$. Region IV of Fig. 2b is similar to the intrinsic region in Fig. 1b. When the temperature dependence of the majority carrier densities in Fig. 2b is compared with that of Fig. 1b, the compensated system reaches the intrinsic region faster than the un-compensated system.

As can be seen in Fig. 2c, the chemical potential corresponding to a given acceptor/donor level lies below/above the intrinsic level when the ionization density corresponding to the acceptor/donor level is less than the intrinsic density and vice versa. One can also see from relation (14) that the low-temperature chemical potential corresponding to a given shallow acceptor/donor level approaches the valence/conduction band edge. Since the majority carrier density in highly compensated p-type semiconductor is less than that of the non-compensated system with equal acceptor density and activation energy, the chemical potential, $E_{\mu \mathrm{a}}$, corresponding to the acceptor level of the highly compensated system lies below the Fermi-energy level, $E_{\mathrm{F}}$, in the extrinsic temperature region as shown in Fig. 2c. The curves of $E_{\mu \mathrm{a}}$ and $E_{\mu \mathrm{d}}$ for a highly compensated system also cross each other on the curve of the intrinsic energy level, $E_{\mathrm{i}}$. The Fermi-energy level, $E_{\mathrm{F}}$, is dominated by the intrinsic energy level, $E_{\mathrm{i}}$, in the intrinsic region.

\section{System with two acceptor levels}

For a system with more than one acceptor level, the net ionization density of the system is given by the sum of the ionization densities of the individual acceptors as given in relation (6). Figure 3 depicts the temperature dependence of (a) the shallow (cyan line), the deep (magenta line) and the net (red line) acceptor ionizations, (b) the majority (wine line), the minority (blue line) and the intrinsic (yellow line) carrier concentrations along with the net acceptor ionizations (red line) and (c) the energy band structure consisting of $E_{\mathrm{V}}$ and $E_{\mathrm{C}}$ (black lines), $E_{\mathrm{F}}$ (wine line), $E_{\mathrm{i}}$ (yellow line), shallow level acceptor energy, $E_{\mu \mathrm{a}}$ (cyan line) and deep level acceptor energy, $E_{\mu \mathrm{a} 2}$ (magenta line) for GaSb sample with two acceptor levels of densities $N_{\mathrm{a} 1}=10^{16} \mathrm{~cm}^{-3}$ and $N_{\mathrm{a} 2}=10^{17} \mathrm{~cm}^{-3}$ and the corresponding activation energies of $E_{\mathrm{a} 1}=1 \mathrm{meV}$ and $E_{\mathrm{a} 2}=200 \mathrm{meV}$. The net ionization density is obtained by the addition of two ionization density of the two acceptors as given in relation (11). The majority carrier density, $p_{0}$, is described using relation (7) by taking the net ionization, $N_{\mathrm{a}}^{-}=N_{\mathrm{a} 1}^{-}+N_{\mathrm{a} 2}^{-}$, into account. The chemical potentials corresponding to shallow and the deep acceptors, $E_{\mu \mathrm{a} 1}$ and $E_{\mu \mathrm{a} 2}$, are treated independently for each acceptor ionizations using relation (12). The Fermi-energy level is described using relation (1).

As can be seen from Fig. 3a, a shallow acceptor with lower density dominates the net ionization in the lowertemperature regime and a deep acceptor can dominate the net ionization in the higher-temperature regime if its ionization density is larger than that of the shallow acceptor. The deep acceptor has less or no effect on the net ionization density if its ionization density is less than that of the shallow level. Since activation energy of the acceptor has no effect in the higher-temperature regime, acceptors with different activation energies and equal densities have the same effect in the complete ionization region (Region II) and the shallow level is dominant in the partial ionization region (Region I). The width of the partial ionization region (Region I) increases with increasing the dopant density and the deepness of the acceptor level as shown in Fig. 3a. The majority carrier density is dominated by the shallow acceptor ionization density at very low temperature regimes and with the deep acceptor level in the intermediate-temperature regions as shown in Fig. 3b. The very high temperature majority carrier is dominated by the intrinsic carrier density.

As depicted in Fig. 3c, the chemical potential corresponding to the shallow acceptor is less than that of the deep level acceptor. The gap between the valence band and the chemical potential also remains constant at half of the acceptor ionization energy $\left(E_{\mu a 1}=0.5 \mathrm{meV}\right.$ and $E_{\mu \mathrm{a} 2}=100 \mathrm{meV}$ ) when evaluated near the absolute zero as predicted in relation (14). The decrease in the slopes of the curves of the chemical potentials with decreasing temperature is the proof for the premise that the chemical potentials are independent of temperature at very low temperature regimes. The chemical potential corresponding to the acceptor with high dopant density is less than that with relatively low dopant density in the higher-temperature regimes as predicted by relation (14). Therefore, the Fermi-energy level, $E_{\mathrm{F}}$, is dominated by the chemical potential corresponding to the shallow level in 

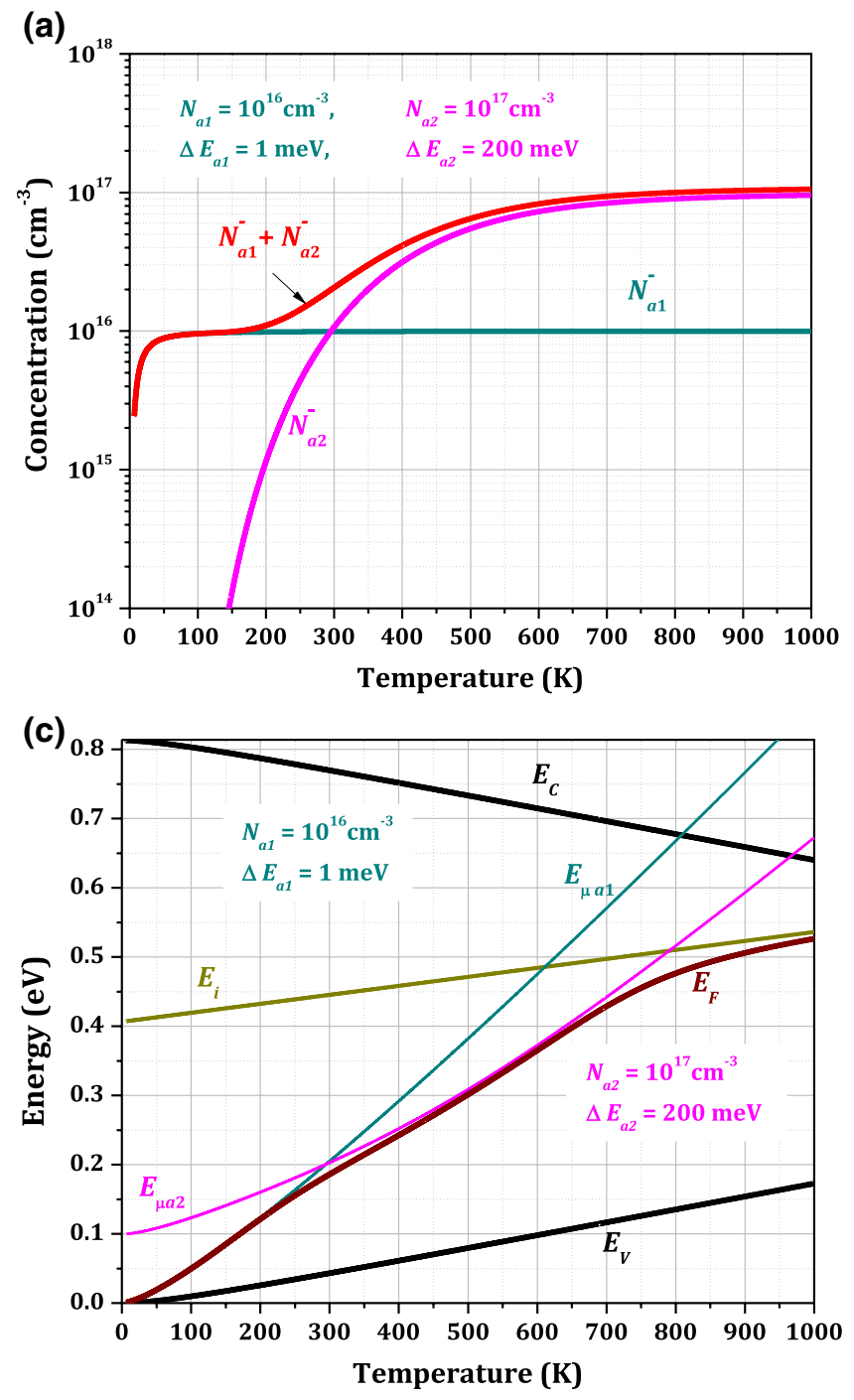

Fig. 3 Temperature dependence of a the shallow (cyan line), the deep (magenta line) and the net (red line) acceptor ionizations, $\mathbf{b}$ the majority (wine line), the minority (blue line) and the intrinsic (yellow line) carrier concentrations along with the net acceptor ionizations (red line); and $\mathbf{c}$ the energy band structure consisting of $E_{\mathrm{V}}$ and $E_{\mathrm{C}}$

the lower-temperature regime, by the chemical potential corresponding to the level with high acceptor density in the intermediate-temperature regime and by the intrinsic energy level, $E_{\mathrm{i}}$, in the higher-temperature regime as depicted in Fig. 3c.

\section{Fitting of the theoretical to the experimental values}

As already discussed in the previous two subsections, the effects of a dopant with very low density on the majority carrier density can be detected if it is shallower than the other dopants. In general, four dopant levels were identified in all of the three samples of GaSb used in this work: two deep acceptors with very large densities and dominating the

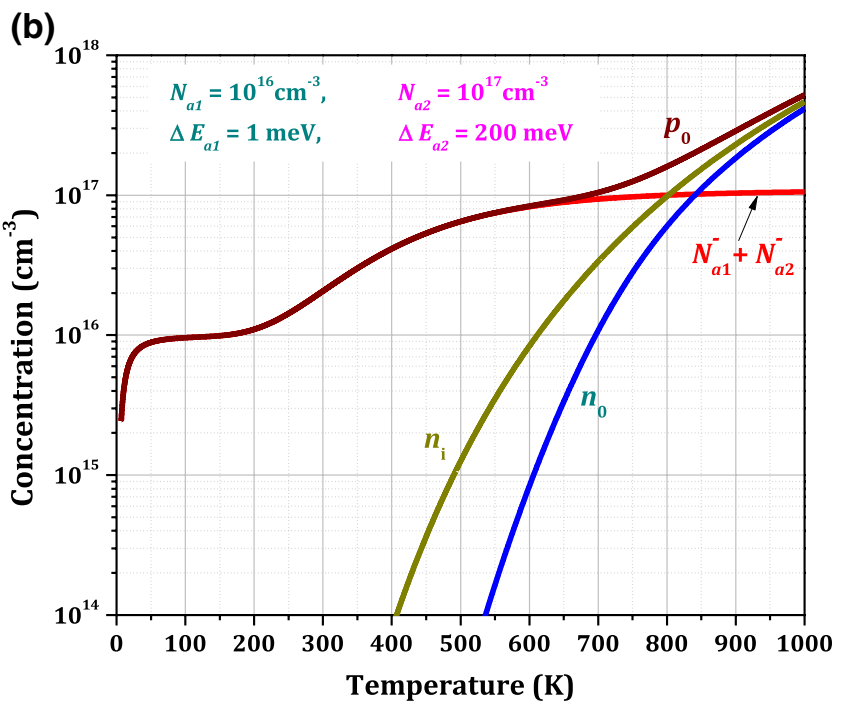

(black lines), $E_{\mathrm{F}}$ (wine line), $E_{\mathrm{i}}$ (yellow line), shallow level acceptor energy, $E_{\mu \mathrm{a} 1}$ (cyan line) and deep level acceptor energy, $E_{\mu \mathrm{a} 2}$ (magenta line) for a semiconductor with two acceptor levels of densities of $N_{\mathrm{a} 1}=10^{16} \mathrm{~cm}^{-3}$ and $N_{\mathrm{a} 2}=10^{17} \mathrm{~cm}^{-3}$ and the corresponding activation energies of $\Delta E_{\mathrm{a} 1}=1 \mathrm{meV}$ and $\Delta E_{\mathrm{a} 2}=200 \mathrm{meV}$

majority carrier densities in the intermediate-temperature region below the quasi-intrinsic region; and one very shallow acceptor with very low density compensated by very shallow and one low-density donor dominating the majority carrier density at very low temperature region. Figure 4 depicts the temperature dependence of the theoretical majority carrier densities, $p_{0}$ (wine lines) fitted to the experimental results of the majority carrier densities (open wine circles), two deep acceptors (cyan and magenta lines), one shallow acceptor (green line) and one donor (green line) detected in the three different p-type GaSb samples labelled (a) S1, (b) S2 and (c) S3. As can be observed from Fig. 4a-c, two hills and one valley are observed in the experimental curves. Two smooth small bumps are also observed approximately 

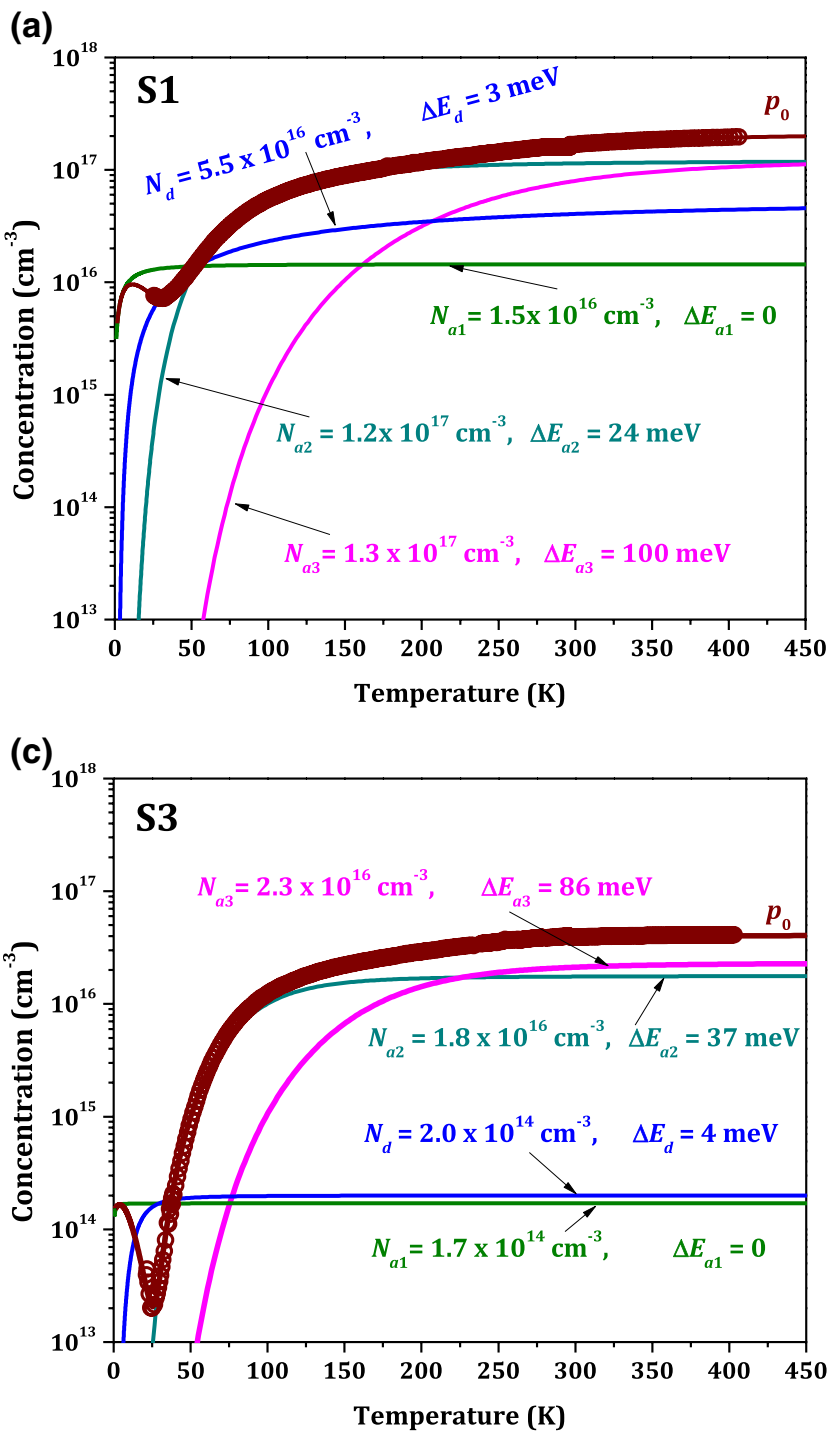

Fig. 4 Temperature dependence of the theoretical majority carrier densities (wine lines) fitted to the experimental results of the majority carrier densities (open wine circles), two deep acceptors (cyan

around $100 \mathrm{~K}$ and $270 \mathrm{~K}$ on the top of the large plateaus at the right sides of the valleys. The curves with negative slopes at lower-temperature regime on the left side of the valleys in all the experimental curves in Fig. $4 \mathrm{a}-\mathrm{c}$ are fitted using the method used in "System with two acceptor levels" section, with the very shallow and low-density acceptors (density, $N_{\mathrm{a} 1}$, and activation energy, $\Delta E_{\mathrm{a} 1}$ ) compensated by the very shallow and low-density donors (density, $N_{\mathrm{d}}$, and activation energy, $\Delta E_{\mathrm{d}}$ ). The smooth bumps on the plateaus on the right sides of the valleys in Fig. $4 \mathrm{a}-\mathrm{c}$ are also fitted with two acceptors (one relatively shallow and the other deep with activation energies $\Delta E_{\mathrm{a} 2}$ and $\Delta E_{\mathrm{a} 3}$, respectively) with comparable densities represented by $N_{\mathrm{a} 2}$ and $N_{\mathrm{a} 3}$ using the method used in "Highly compensated system" section. The entire net ionization densities of the system are

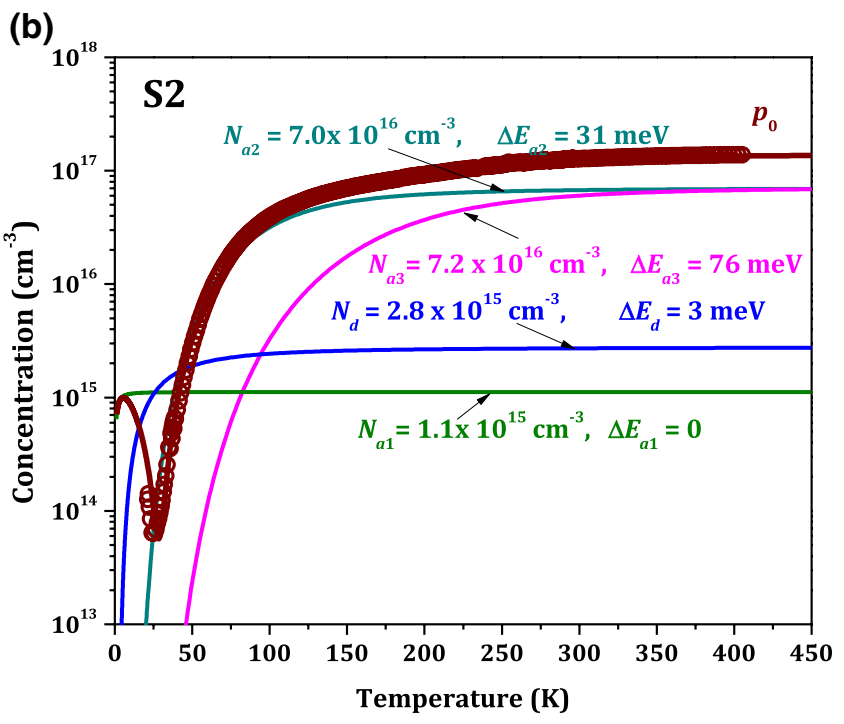

and magenta lines), one shallow acceptor (green line) and one donor (green line) detected in the three different p-type GaSb samples labelled a S1, b S2 and c S3

determined by subtracting the ionization densities of the acceptors from the net (summation of) ionization densities of the three acceptors, as discussed in relations (6) and (7). Fitting can be done by adjusting the values of the dopant densities and the activation energies until the theoretical values of $p_{0}$ in relation (7) exactly fit the experimental values as shown in Fig. $4 \mathrm{a}-\mathrm{c}$.

The very shallow donors detected in the three samples have activation energies $3 \mathrm{meV}, 3 \mathrm{meV}$ and $4 \mathrm{meV}$ and densities $5.5 \times 10^{16} \mathrm{~cm}^{-3}, 2.8 \times 10^{15} \mathrm{~cm}^{-3}$ and $2.0 \times 10^{14} \mathrm{~cm}^{-3}$, respectively. The respective very shallow acceptors involved in the compensation process along the very shallow donors in these samples have zero activation energies and densities $1.5 \times 10^{16} \mathrm{~cm}^{-3}, 1.1 \times 10^{15} \mathrm{~cm}^{-3}$ and $1.7 \times 10^{14} \mathrm{~cm}^{-3}$. The very deepest acceptors detected in the samples have 
activation energies $100 \mathrm{meV}, 76 \mathrm{meV}$ and $86 \mathrm{meV}$ and densities $1.3 \times 10^{17} \mathrm{~cm}^{-3}, 7.2 \times 10^{16} \mathrm{~cm}^{-3}$ and $2.3 \times 10^{16} \mathrm{~cm}^{-3}$, respectively. The other respective deep acceptors detected have activation energies $24 \mathrm{meV}, 31 \mathrm{meV}$ and $37 \mathrm{meV}$ and densities $1.2 \times 10^{17} \mathrm{~cm}^{-3}, 7.0 \times 10^{16} \mathrm{~cm}^{-3}$ and $1.8 \times 10^{16} \mathrm{~cm}^{-3}$. The last acceptor levels obtained are in complete agreement with the dominant residual acceptor levels of activation energies $20 \mathrm{meV}$ to $40 \mathrm{meV}$ and densities $10^{16} \mathrm{~cm}^{-3}$ to $10^{18} \mathrm{~cm}^{-3}$ reported by Ling et al. and others $[1,2,8,16]$. The two dominant deep acceptors reported by Matsuura et al. and others also confirm the result obtained [9-11, 13]. The possibility of self-compensation reported by several writers is also the confirmation of the obtained results $[9-12,15]$.

(a)
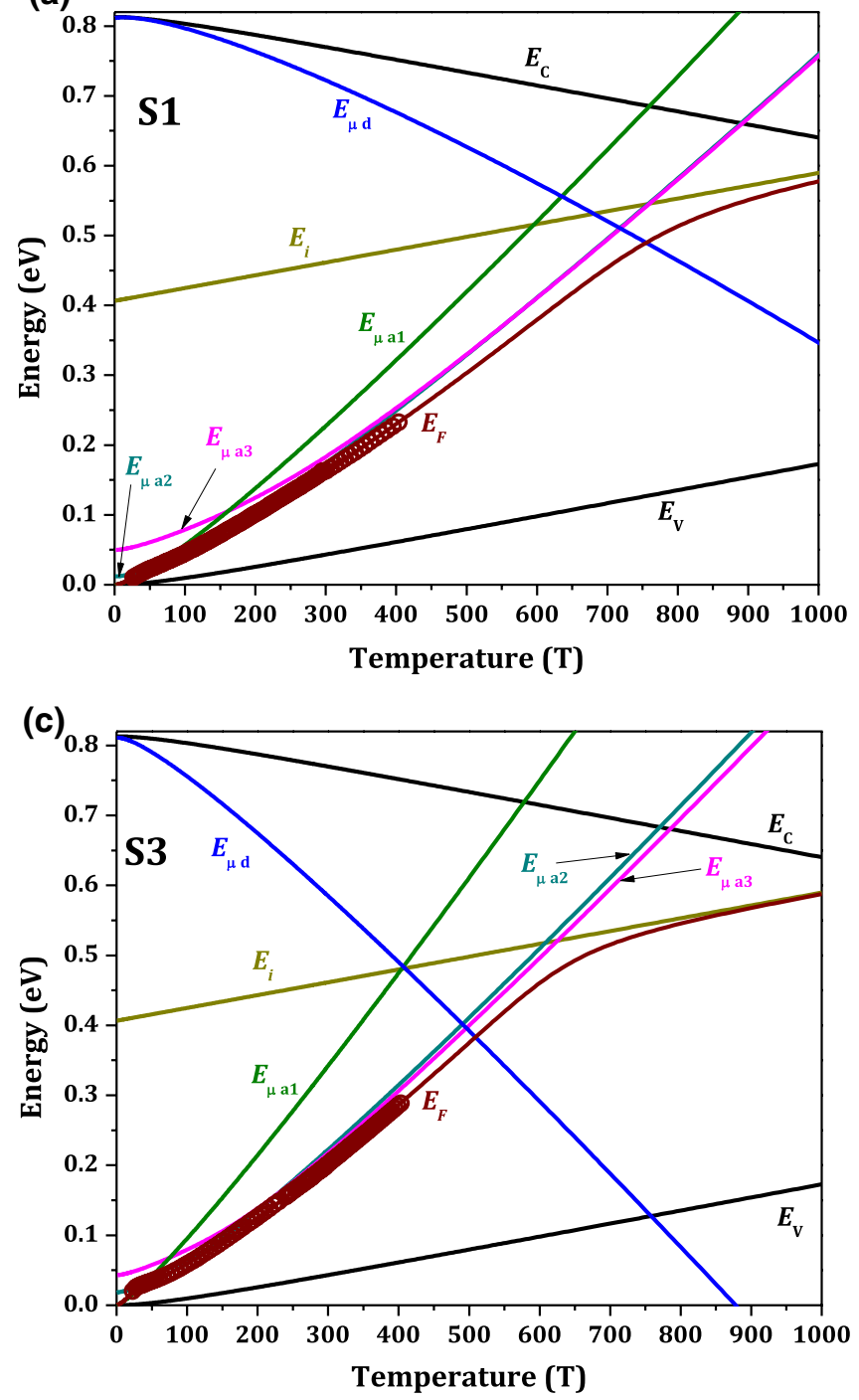

The compensation effects of the shallow acceptors and donors with very low densities are detected only in the very low temperature regions as shown in Fig. $4 \mathrm{a}-\mathrm{c}$. This effect is suppressed by the effects of the two deep acceptors with high densities in the intermediate-temperature region. Using this method, one can detect only the very shallowest and densest dopants. The involvement of the deepest and less density donors in the self-compensation reported by Hubik et al. $[9,10]$ in tellurium- and sulphur-doped GaSb cannot be detected using this method.

Figure 5 illustrates the temperature dependence of the energy band structures of the respective samples discussed in Fig. 4. The energy band structure consists of $E_{\mathrm{V}}$ and $E_{\mathrm{C}}$ (black lines), $E_{\mathrm{F}}$ (wine line), $E_{\mathrm{i}}$ (yellow line), the chemical potentials corresponding to the three acceptors $E_{\mu \mathrm{a} 1}, E_{\mu \mathrm{a} 2}$ and

(b)

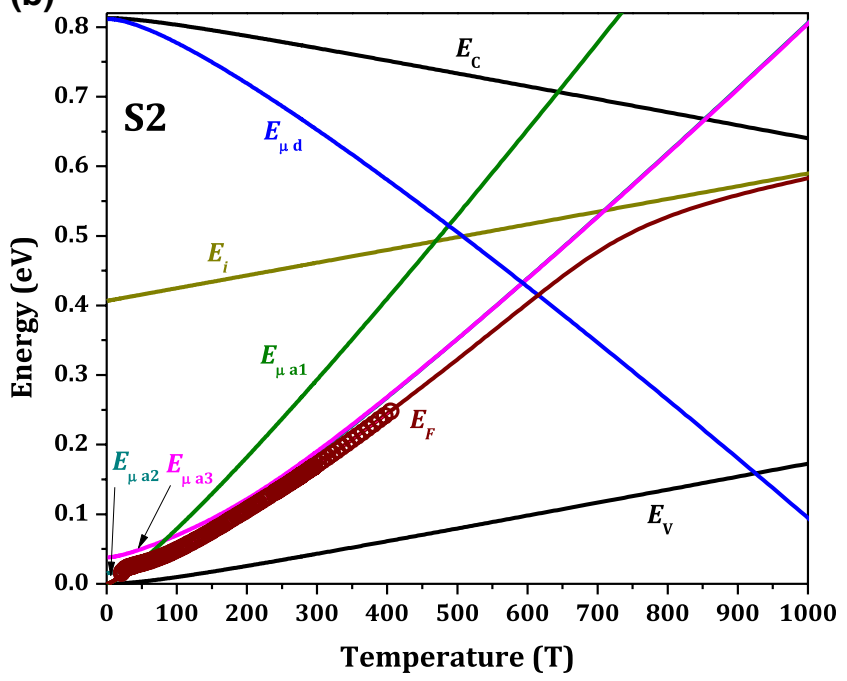

Fig. 5 Temperature dependence of the energy band structures of the respective samples detected in the three different p-type GaSb samples labelled a S1, b S2 and c S3 discussed in Fig. 4, respectively 
$E_{\text {на3 }}$ (green, cyan, magenta lines) and that corresponding to the shallow donor $E_{\mu \mathrm{d}}$ (blue line). The temperature dependence of $E_{\mu \mathrm{a} 1}, E_{\mu \mathrm{a} 2}$ and $E_{\mu \mathrm{a} 2}$ are determined independently by varying the respective acceptor densities, $N_{\mathrm{a} 1}, N_{\mathrm{a} 2}$ and $N_{\mathrm{a} 3}$, the acceptor activation energies $\Delta E_{\mathrm{a} 1}, \Delta E_{\mathrm{a} 2}$ and $\Delta E_{\mathrm{a} 3}$ and the temperature $T$ using relation (12). The experimental values of the Fermi-energy levels, $E_{F}$ labelled by the wine open circles in Fig. 5a-c were determined using the experimental results in Fig. 4a-c along with relation (1).

One can see from Fig. 5a-c that the chemical potentials corresponding to the shallowest acceptor, $E_{\mu \mathrm{a} 1}$, dominate the Fermi-energy level in the lowest temperature regimes. Since the densities of the two deep acceptors in a sample are nearly equal, the chemical potentials corresponding to these acceptors dominate the Fermi-energy level equally in the intermediate-temperature ranges below the quasi-intrinsic region. The chemical potentials corresponding to the very deep acceptors and donors with very lower majority carrier densities are going deep into the band gap as compared to those of dopants with very high majority carrier densities.

\section{Conclusion}

It was a long-established fact for a semiconductor with a single dopant level that in the extrinsic temperature ranges below the quasi-intrinsic temperature region, the dopant ionization density dominates the majority carrier density, and hence the chemical potential corresponding to the dopant level dominates the entire Fermi-energy level below the quasi-intrinsic temperature region. Upon equating the expression of the majority carrier density to that of the dopant ionization density, the expression for the chemical potential corresponding to the dopant level was established in terms of the dopant density, the activation energy of the dopant and the effective density of the respective band states. Again, upon substituting the Fermi-energy level in the expression of the majority carrier density by the chemical potential in the expression of the dopant ionization density, the expression for the dopant ionization density was established in terms of the dopant density, the activation energy of the dopant and the effective density of the respective band states. The established facts were evaluated for one-acceptorlevel system, highly compensated system and two-acceptorlevel system with the help of illustrations.

The dopant ionization densities and the majority carrier density increase with increasing temperature, decrease with increasing the dopant activation energies in the lower-temperature regime and are unaffected by both the temperature and the dopant activation energies in the higher-temperature regime. The deepness of the chemical potentials corresponding to the dopant levels increases by one half of the activation energy with increasing the deepness of the activation energy and unaffected by the temperature and the dopant densities at very low temperature regimes. The chemical potentials corresponding to the dopant levels increase with increasing the temperature and decreasing the dopant densities and are unaffected by the dopant activation energies in the higher-temperature regimes. The established facts were well corroborated by the fitting of the theoretical to the experimental results of the majority carrier densities obtained by the temperature-dependent Hall effect measurements. From the fitting of the theoretical to the experimental results, four dopant levels are detected in p-type $\mathrm{GaSb}$. The very shallow acceptor and donor with densities $10^{14}-10^{16} \mathrm{~cm}^{-3}$ and energies $0-4 \mathrm{meV}$ are detected at very low temperature region. The two relatively deep acceptors with activation energies $20-40 \mathrm{meV}$ and $75-100 \mathrm{meV}$ and densities $10^{16}-10^{17} \mathrm{~cm}^{-3}$ are also detected at intermediate temperature below the quasi-intrinsic region.

Open Access This article is distributed under the terms of the Creative Commons Attribution 4.0 International License (http://creativeco mmons.org/licenses/by/4.0/), which permits unrestricted use, distribution, and reproduction in any medium, provided you give appropriate credit to the original author(s) and the source, provide a link to the Creative Commons license, and indicate if changes were made.

\section{References}

1. Ling, C.C., Mui, W.K., Lam, C.H., Beling, C.D., Fung, S., Lui, M.K., Cheah, K.W., Li, K.F., Zhao, Y.W., Gong, M.: Gallium vacancy and the residual acceptor in undoped GaSb studied by positron lifetime spectroscopy and photoluminescence. Appl. Phys. Lett. 80, 3934-3936 (2002)

2. Hakala, M., Puska, M.J., Nieminen, R.M.: Native defects and selfdiffusion in GaSb. J. Appl. Phys. 91, 4988-4994 (2002)

3. Rogalski, A.: Quantum well photoconductors in infrared detector technology. Appl. Phys. Rev. 93, 4355-4391 (2003)

4. Joullié, A.F., Christola, P., Rodriguez, J.B., Ait-Kaci, H., Chevrier, F., Nieto, J., De Anda, F.: A survey of new laser and detector structures for 3-5 $\mu \mathrm{m}$ midinfrared spectral range. Adv. Optoelectron. Lasers 211, 5582 (2004)

5. Streetman, B.G., Banerjee, S.K.: Solid State Electronic Devices, 6th edn. Asoke K. Ghosh, PHI Learning P. L., New Delhi (2009)

6. Perriet, R.F.: Advanced Semiconductor Fundamentals, 2nd edn. Prentice Hall, Upper Saddle River (2004)

7. Li, S.S.: Semiconductor Physical Electronics, vol. 45, pp. 63736375. Springer, New York (2006)

8. Ling, C.C., Lui, M.K., Ma, S.K., Chen, X.D., Fung, S., Beling, C.D.: Nature of the acceptor responsible for p-type conduction in liquid encapsulated Czochralski-grown undoped gallium antimonide. Appl. Phys. Lett. 85, 384-386 (2004)

9. Hubik, P., Mares, J.J., Kristofik, J., Baraldi, A., Ghezzi, C., Parisini, A.: Hall and photo-Hall effect measurements on sulphurdoped GaSb. Semicond. Sci. Technol. 10, 455-462 (1995)

10. Hubik, P., Mares, J.J., Kristofik, J., Sestakova, V., Stepanek, B.: Deep donors in tellurium and sulphur codoped GaSb. Semicond. Sci. Technol. 11, 989-995 (1996)

11. Dutta, P.S., Prasad, V., Bhat, H.L.: Carrier compensation and scattering mechanisms in p-GaSb. J. Appl. Phys. 80, 2847-2853 (1996) 
12. Nakashima, K.: Electrical and optical studies in GaSb. Jpn. J. Appl. Phys. 20, 1085 (1981)

13. Matsuura, H., Morita, K., Nishikawa, K., Mizukoshi, T., Segawa, M., Susaki, W.: Acceptor densities and acceptor levels in undoped GaSb determined by free carrier concentration spectroscopy. Jpn. J. Appl. Phys. 41, 496-500 (2002)

14. Mathur, P.C., Jain, S.: Hole transport properties in gallium antimonide from 77 to 300 K. Phys. Rev. B 19, 3152-3158 (1977)

15. Zhao, G.Y., Ebisu, H., Soga, T., Egawa, T., Jimbo, T., Umeno, N.: Electrical transport properties of GaSb grown by molecular beam epitaxy. Jpn. J. Appl. Phys. 1, 1704-1708 (1998)

16. Pödör, B., Somogyi, K.: Thermal activation energy of natural acceptors in GaSb. In: International Conference of Microelectronics (SPIE), Warsaw (1992)
17. Blakemore, J.S.: Semiconductor Statistics. Dover Publications, Inc., New York (1987)

18. Blakemore, J.S.: Solid state physics, 2nd edn. Cambridge University Press, New York (1998)

19. Varshni, V.P.: Band-to-band radiative recombination in groups IV, VI, and III-V semiconductors. Physica Status Solidi 19, 459-514 (1967)

Publisher's Note Springer Nature remains neutral with regard to jurisdictional claims in published maps and institutional affiliations. 